\title{
Drug-Drug Compatibility Evaluation of Sulfasalazine and Folic Acid for Fixed-Dose Combination Development Using Various Analytical Tools
}

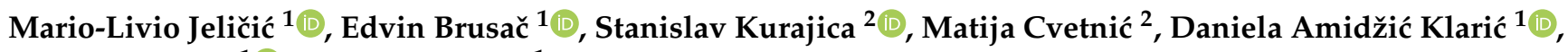 \\ Biljana Nigović ${ }^{1}\left(\mathbb{D}\right.$ and Ana Mornar ${ }^{1, *}$ \\ 1 Faculty of Pharmacy and Biochemistry, University of Zagreb, A. Kovačića 1, 10000 Zagreb, Croatia; \\ mljelicic@pharma.hr (M.-L.J.); ebrusac@pharma.hr (E.B.); damidzic@pharma.hr (D.A.K.); \\ bnigovic@pharma.hr (B.N.) \\ 2 Faculty of Chemical Engineering and Technology, University of Zagreb, Marulić Square 19, \\ 10000 Zagreb, Croatia; stankok@fkit.hr (S.K.); mcvetnic@fkit.hr (M.C.) \\ * Correspondence: amornar@pharma.hr; Tel.: +38-514-818-288
}

\section{check for} updates

Citation: Jeličić, M.-L.; Brusač, E.; Kurajica, S.; Cvetnić, M.; Amidžić Klarić, D.; Nigović, B.; Mornar, A. Drug-Drug Compatibility Evaluation of Sulfasalazine and Folic Acid for Fixed-Dose Combination Development Using Various Analytical Tools. Pharmaceutics 2021, 13, 400. https://doi.org/10.3390/ pharmaceutics 13030400

Academic Editor: Beverley D. Glass

Received: 26 February 2021

Accepted: 16 March 2021

Published: 17 March 2021

Publisher's Note: MDPI stays neutral with regard to jurisdictional claims in published maps and institutional affiliations.

Copyright: (c) 2021 by the authors. Licensee MDPI, Basel, Switzerland. This article is an open access article distributed under the terms and conditions of the Creative Commons Attribution (CC BY) license (https:/ / creativecommons.org/licenses/by/ $4.0 /)$.

\begin{abstract}
The simultaneous administration of sulfasalazine and folic acid is regular practice in the therapy of inflammatory bowel diseases in order to maintain sufficient folate concentration in patients. Having multiple drugs in the therapy increases the possibility of patients failing adherence, thus unintentionally endangering their health. A fixed-dose combination of sulfasalazine and folic would simplify the classical polytherapeutic approach; however, the physicochemical compatibility investigation of two active pharmaceutical ingredients plays an important role in the development of such a product. In this work, various analytical tools were used to determine the physicochemical compatibility of sulfasalazine and folic acid. For the evaluation of chemical compatibility, infrared spectroscopy in combination with advanced statistical methods, such as the principal component analysis and cluster analysis, were used, whilst a simultaneous thermogravimetry/differential thermal analysis gave us an insight into the physical compatibility of two drugs. Isothermal stress testing, forced degradation and dissolution studies, followed by the analysis with a developed chromatographic method for the monitoring of folic acid, sulfasalazine and two of its related impurities, sulfapyridine and salicylic acid, gave us an insight into its chemical compatibility. The combination of the results obtained from the used techniques implies a satisfactory physicochemical compatibility between sulfasalazine and folic acid, which opens the path to the development of the proposed fixed-dose combination.
\end{abstract}

Keywords: sulfasalazine; folic acid; fixed-dose combination; physicochemical compatibility; thermal techniques; spectroscopy; chromatography

\section{Introduction}

Sulfasalazine (SASP) is an anti-inflammatory drug that has been mainly used for the treatment of rheumatoid arthritis since its discovery in the 1930s. Nevertheless, patients with ulcerative colitis given the SASP therapy have noticed a significant reduction of symptoms, which ultimately led to its use in the treatment of inflammatory bowel diseases (IBD) [1]. The SASP molecule consists of the anti-inflammatory 5-aminosalicylic acid and an antibiotic sulfapyridine (SP) connected via an azo bond. Once administered, SASP is absorbed up to $25 \%$ in the small intestine, while the rest of the drug reaches the colon mostly unchanged, where enzyme azo-reductase breaks the azo bond, releasing 5-aminosalicylic acid at the site of inflammation [2]. The main downsides of SASP are the toxicity of its metabolite SP, which is responsible for its side effects in nearly $30 \%$ of patients, and the SASP-induced inhibition of the reduced folate carrier at clinically relevant plasma concentrations. This inhibition leads to a folate deficiency in patients that consequently 
increases the chances of developing colorectal cancer and megaloblastic anemia, as well as defects, in newborns [3-6]. To maintain a sufficient folate concentration in IBD patients, a supplementation of folic acid (FA) during the regular therapy of IBD is mandatory, usually in elevated doses of $1-5 \mathrm{mg} /$ day compared to the intake of $0.4 \mathrm{mg} /$ day in healthy people [7].

A fixed-dose combination (FDC), a drug formulation comprising of multiple active pharmaceutical ingredients (APIs), is a great alternative to the classical therapy approach. For diseases that require polytherapy, such as IBD, multiple drugs must be administered simultaneously, increasing the risk that some of the medications will be omitted. SASP/FA FDC would increase the medication adherence in IBD patients by ensuring the regular intake of FA, where the proposed FDC would contain enough SASP and FA to maintain the remission state of the disease and to supplement malabsorbed FA during the therapy. The process of FDC development requires thorough drug-drug compatibility testing to ensure the physical and chemical compatibility of APIs. Physical and chemical incompatibility can result in unwanted interactions between two drugs leading to the questionable safety, efficacy and stability of the final product. Physical incompatibility can result in the change of odor, color and morphology whilst chemical incompatibility can lead to the creation of new inactive or toxic products [8]. Various techniques and methods, such as Fourier-transform infrared spectroscopy (FTIR), simultaneous thermogravimetry, differential thermal analysis (TG/DTA) and isothermal stress testing (IST), have been used so far for examining drug-drug and drug-excipient physicochemical compatibility [9-12]. Forced degradation studies, comprising of a series of degradation conditions applied to APIs and finished products, as well as their blends, are recommended during the FDC development process by The World Health Organisation guidelines for the registration of FDCs [13].

Up until now, there has been no published study concerning the physicochemical compatibility investigation of SASP and FA when present in the same solution or blend. In this research, the physicochemical compatibility of SASP and FA was examined using the FTIR, TG/DTA and IST techniques, as well as forced degradation and dissolution studies. For the assay determination after the IST, forced degradations and dissolution studies, a single high-performance liquid chromatography (HPLC) assay method was developed for the monitoring of SASP, FA and two SASP-related impurities, SP and salicylic acid (SA) (Figure 1).<smiles>O=C(O)c1cc(N=Nc2ccc(S(=O)(=O)Nc3ccccn3)cc2)ccc1O</smiles>

a)<smiles>Nc1nc2ncc(CNc3ccc(C(=O)NC(CCC(=O)O)C(=O)O)cc3)nc2c(=O)[nH]1</smiles>

b)<smiles>Nc1ccc(S(=O)(=O)Nc2ccccn2)cc1</smiles><smiles>O=C(O)c1ccccc1O</smiles>

c) d)

Figure 1. Structures of (a) sulfasalazine (SASP), (b) folic acid (FA), (c) sulfapyridine (SP) and (d) salicylic acid (SA). 


\section{Materials and Methods}

\subsection{Reagents and Chemicals}

The SASP (as per the British Pharmacopoeia (BP), 99.6\%), FA (meets the United States Pharmacopoeia (USP) specifications, 100.6\%), SP $(98.0 \%)$ and SA ( $\geq 99.0 \%)$ standards were provided by Sigma-Aldrich (St. Louis, MO, USA). Chemicals used for the preparation of samples, buffers, mobile phase, $\mathrm{pH}$ adjustment and degradation studies were the following: ultrapure water $(<0.1 \mu \mathrm{S} / \mathrm{cm})$ purified with a Series Ultra Clear and Integra Ultra-pure water system (SG Water, Barsbuttel, Germany), methanol and acetonitrile HPLC grade solvents ( $\geq 99.9 \%$ ) (Avantor, Deventer, The Netherlands), $N, N$ dimethylformamide (DMF) (99.5\%) (VWR Chemicals, Radnor, PA, USA), formic acid for HPLC (98-100\%) (Merck, Kenilworth, NJ, USA), glacial acetic acid (99.5-100.5\%) (Panreac Química S.L.U., Barcelona, Spain), ammonium acetate ( $\geq 98.0 \%)$ (Lach-Ner s.r.o., Neratovice, Czech Republic), sodium hydroxide pellets ( $\geq 98.0 \%$ ) (Sigma-Aldrich), hydrochloric acid (37.0\%) (Carlo Erba, Val-de-Reuil, France), hydrogen peroxide ( $\geq 30.0 \%)$ (T.T.T. d.o.o., Sveta Nedelja, Croatia), monobasic potassium phosphate $(\geq 99.0 \%)$ (Kemika, Zagreb, Croatia) and $\alpha$-alumina (Sigma-Aldrich).

Finished drug products Sulfasalazin Krka EN 500-mg gastro-resistant tablets (KRKA, d.d., Novo Mesto, Slovenia) and Folacin ${ }^{\circledR}$ 5-mg tablets (Jadran-Galenski Laboratorij d.d., Rijeka, Croatia) used in experiments were obtained from the local pharmacy. For selectivity tests and method validation, the following excipients (present in the above-stated drug products) were used for the preparation of the placebo blend: lactose monohydrate, cellulose (microcrystalline), povidone, crospovidone, starch, magnesium stearate and silicon dioxide (colloidal anhydrous). All excipients were obtained from Fagron Hrvatska d.o.o. (Donja Zelina, Croatia).

\subsection{Preparation of Working Solutions and Blends}

Stock solution containing 1000- $\mu \mathrm{g} / \mathrm{mL}$ SASP, $2-\mu \mathrm{g} / \mathrm{mL}$ FA, $5-\mu \mathrm{g} / \mathrm{mL}$ SP and $5-\mu \mathrm{g} / \mathrm{mL}$ SA was prepared by carefully weighing and dissolving each standard in $30 \%$ DMF. Working solution, containing $250-\mu \mathrm{g} / \mathrm{mL}$ SASP, $0.50-\mu \mathrm{g} / \mathrm{mL}$ FA, $1.25-\mu \mathrm{g} / \mathrm{mL}$ SP and $1.25-\mu \mathrm{g} / \mathrm{mL}$ SA, was prepared by diluting the stock solution with $30 \%$ DMF and the addition of the placebo blend followed by 10-min sonication in an ultrasonic bath (Elmasonic XtraTT, Biosan, Riga, Latvia). Working solution contained the maximum allowed concentration of impurities (in relation to SASP), as per the BP monograph for the SASP finished product [14]. Prior to HPLC analysis, the working solution was centrifuged and filtered through a polyethersulfone (PES) syringe filter $(25 \mathrm{~mm}, 0.22 \mu \mathrm{m})$ (Obrnuta faza d.o.o., Pazin, Croatia) to remove the residues of undissolved excipients.

Standard and tablet blends for IST tests and forced degradation studies were prepared in the proposed 500:1 ratio by weighing (using a MX5 microbalance scale by Mettler Toledo, Columbus, OH, USA) and mixing $2000 \mathrm{mg}$ of SASP and $4 \mathrm{mg}$ of FA standard or, in the case of tablet blend preparation, by weighing the amount of previously powdered tablets equivalent to $2000 \mathrm{mg}$ of SASP and $4 \mathrm{mg}$ of FA. Standard blends for TG/DTA measurements were prepared in a 1:1 ratio by mixing equal amounts of each standard, whilst, for the FTIR measurements, 500:1, 10:1, 5:1, 2:1, 1:1, 1:2, 1:5 and 1:10 blends were prepared. Placebo blend was prepared by mixing the commonly used excipients in a ratio that represented their maximum allowed concentration in the formulation [15]. All powders were thoroughly mixed using mortar and pestle for $20 \mathrm{~min}$ to secure complete homogenization.

\subsection{Chromatographic Conditions}

Agilent 1100 series HPLC system (Agilent Technologies, Waldbronn, Germany) coupled with a diode array detector with Chemstation software for data processing was used for all chromatographic analyses. Separation was performed on XBridge C18 $(150 \times 4.6 \mathrm{~mm}$, 3.5- $\mu \mathrm{m}$ particle size) reverse-phase column with suitable guard column, both obtained by Waters (Milford, MA, USA). The column was thermostated at $25.0 \pm 0.1{ }^{\circ} \mathrm{C}$ during the analysis, with a constant flow rate of $1.0 \mathrm{~mL} / \mathrm{min}$. The mobile phase consisted of $5.0-\mathrm{mM}$ 
ammonium acetate buffer ( $\mathrm{pH}$ set to $5.00 \pm 0.01$ using a FiveEasy $\mathrm{pH}$ meter by Mettler Toledo) as mobile phase A and methanol as mobile phase B. Gradient elution program was as follows: 0-8 min, linear gradient 5-40\% B; 8-10 min, linear gradient 40-80\% B; 10-15 min, isocratic $80 \%$ B and 15-18 min, linear gradient $80-100 \%$ B. Analysis time was $18 \mathrm{~min}$, with an additional $7 \mathrm{~min}$ to re-equilibrate the stationary phase for the following analysis. Injection volume of $5.0 \mu \mathrm{L}$ and detection wavelength of $360 \mathrm{~nm}$ was chosen for the monitoring of SASP, whilst FA, SA and SP were monitored at $285 \mathrm{~nm}$ with a $20.0-\mu \mathrm{L}$ injection volume.

\subsection{TG/DTA Analyses, IST Conditions and FTIR Settings}

TG/DTA analyses were carried out using the NETZSCH thermal analyser STA 409 (NETZSCH-Gerätebau GmbH, Selb, Germany). Approximately $10.0 \pm 0.1 \mathrm{mg}$ of the sample was carefully weighed directly into the priorly cleaned platinum pan. Measurements were performed under the atmosphere of synthetic air in a flow rate of $30 \mathrm{~mL} / \mathrm{min}$ from $25^{\circ} \mathrm{C}$ up to $400{ }^{\circ} \mathrm{C}$, with the heating rate of $10^{\circ} \mathrm{C} / \mathrm{min}$ and the usage of $\alpha$-alumina as a reference.

IST was performed on SASP and FA standards, tablets, standard blends and tablet blends. Samples were spread in a thin layer on a Petri dish, visually inspected and placed in the thermostat (ES-20/60 Orbital Shaker-Incubator by Biosan) for 4 weeks at a temperature of $50{ }^{\circ} \mathrm{C}$. After the heating period, samples were examined to identify any visual changes and analyzed using the developed HPLC method and FTIR.

For the chromatographic analysis, $5 \mathrm{mg}$ of stressed sample was weighed and dissolved in $20 \mathrm{~mL}$ of $30 \%$ DMF, centrifuged, filtered and analyzed using the developed method.

Infrared spectra of SASP and FA, as well as all prepared blends, were obtained in the range from 500-4000 $\mathrm{cm}^{-1}$ using a Fourier-transform infrared spectrophotometer FTIR-8400S by Shimadzu (Kyoto, Japan) with PIKE MIRacle ${ }^{\mathrm{TM}}$ universal ATR by PIKE Technologies (Madison, WI, USA). All spectra were obtained as the average of 45 scans with a resolution of $2 \mathrm{~cm}^{-1}$.

\subsection{Forced Degradation Study Conditions}

Forced degradation studies were performed on SASP and FA standards, standards blend and tablets blend. Acid, alkali and oxidative degradation studies were conducted on solutions in $0.1-\mathrm{M} \mathrm{HCl}, 0.1-\mathrm{M} \mathrm{NaOH}$ and $3 \% \mathrm{H}_{2} \mathrm{O}_{2}$. Thermal stability of the solutions and solid samples was examined at a temperature of $70{ }^{\circ} \mathrm{C}$, whilst photostability studies were performed by keeping the samples in indirect daylight. Degradation studies were conducted on solutions containing $250 \mu \mathrm{g} / \mathrm{mL}$ of SASP and $0.5 \mu \mathrm{g} / \mathrm{mL}$ of FA, whilst degradations conducted on solids were performed on accurately weighed amounts of prepared samples that were, after the reaction period, dissolved in 30\% DMF. Samples were kept in described conditions until the optimal degradation of 10-30\% [13]. All samples were analyzed using the developed HPLC method.

\subsection{In Vitro Dissolution Studies}

Dissolution studies of the SASP and FA finished products, as well as their simoultaenous dissolution, were performed as per the USP method for dissolution of SASP delayed-release tablets [16]. The USP 2 dissolution apparatus LDLT-A10 (Labtron Equipment Ltd., Fleet, UK) was used with the paddle rotation speed fixed at $100 \mathrm{rpm}$ and bath thermostated at $37.0^{\circ} \mathrm{C}$. Simulated gastric fluid (SGF; $0.1-\mathrm{N} \mathrm{HCl}$ ) and simulated intestinal fluid (SIF; 0.50-mM phosphate buffer (PB) solution, $\mathrm{pH}$ 7.5) were used as dissolution media at a constant volume of $900 \mathrm{~mL}$ for a two-stage dissolution procedure. The first stage involved the dissolution of tablets in SGF for $120 \mathrm{~min}$, followed by 60 -min dissolution in SIF. During the test, an aliquot of $5 \mathrm{~mL}$ was taken from the dissolution vessel every $30 \mathrm{~min}$ for assay determination using the developed HPLC method. An equal amount of fresh dissolution media was added after each sampling to keep the dissolution media volume constant. Dissolution experiments were conducted in triplicate for each tablet and their combination. 


\subsection{Statistical Methods}

Statistical software XLSTAT by Addinsoft (New York, NY, USA) was used for interpretation of the obtained FTIR spectra and comparison of the results obtained from the chromatographic analysis of samples conducted for IST.

Statistical data processing was performed using the principal component analysis (PCA) with Pearson's correlation and a cluster analysis (CA) using Euclidean distance measures and Ward's agglomerative clustering on the spectral region from $550-1800 \mathrm{~cm}^{-1}$, where most of the information was present. A $10 \times 650$ matrix was created where the number of rows represented SASP, FA and eight of their blends, whilst the columns represented the spectral data obtained with the $2-\mathrm{cm}^{-1}$ resolution.

Two sample $t$-test was used for the comparison of recoveries obtained from the chromatographic analysis of the samples conducted for IST. The first group represented the recoveries of the samples stressed alone, whilst the second were recoveries from the stressed blends.

\section{Results and Discussion}

\subsection{HPLC Method Development and Validation}

The development of the stability-indicating assay method started with the selection of the optimal HPLC column. An XBridge C18 reverse-phase column $(150 \times 4.6 \mathrm{~mm}$, 3.5- $\mu \mathrm{m}$ particle size) with a suitable guard column was used as a starting point for the method development. SASP was expected to be well-retained on the C18 column due to its high $\log K_{\text {ow }}$ value (3.7-4.8) [17]. Using isocratic elution with $50 \%$ methanol acidified with $0.1 \%$ formic acid as the mobile phase resulted in poor resolution between FA, SP and SA $(<1.3)$, whilst SASP was well-retained, however, with prominent peak tailing (3.8). Considering the poor chromatographic performance obtained with the isocratic elution in acidified conditions ( $\mathrm{pH} 2.87$ ), the mobile phase was replaced with 5.0-mM ammonium acetate buffer with the $\mathrm{pH}$ set to $5.00 \pm 0.01$ with acetic acid. A gradient elution was necessary to get acceptable SASP retention and, thus, overall method run time. Usage of the above-defined conditions resulted in the satisfactory separation of all compounds (resolution > 3.98), peak symmetry $(<1.26$, as per USP) and purity factor ( $>998)$ (Figure 2 ).

The column temperature and flow rate had a minor impact on the separation and were set to be constant at $25.0^{\circ} \mathrm{C}$ and $1.0 \mathrm{~mL} / \mathrm{min}$. To increase the sensitivity of the method, FA, SA and SP were monitored at $285 \mathrm{~nm}$ with a $20.0-\mu \mathrm{L}$ injection volume, whilst SASP was monitored at $360 \mathrm{~nm}$ with a $5.0-\mu \mathrm{L}$ injection volume to avoid a column and detector overload.

The method was validated regarding selectivity, linearity, accuracy, precision, limit of quantification, limit of detection and robustness as per the ICH guideline Q2 (R1) [18]. The selectivity of the method was determined using Chemstation software to calculate the peak purity of each peak with the purity factor threshold set at 995 . No interferences were observed at the elution time of the analytes, whilst all peaks were shown to be pure, with the purity factor values greater than 998 . The resolution factor between all the peaks was higher than 3.98, which exceeds the regulatory proposed minimal value of 1.5.

Five-point calibration curves of high linearity $(r \geq 0.999)$ were obtained in the range from $80 \%$ up to $120 \%$ of the working solution concentration for SASP and FA and from the limit of quantification up to $180 \%$ of the maximum allowed impurity concentration for SA and SP as per the BP [14]. The limits of detection and quantification were determined as 3:1 and 10:1 signal-to-noise ratios, respectively (Table 1). 


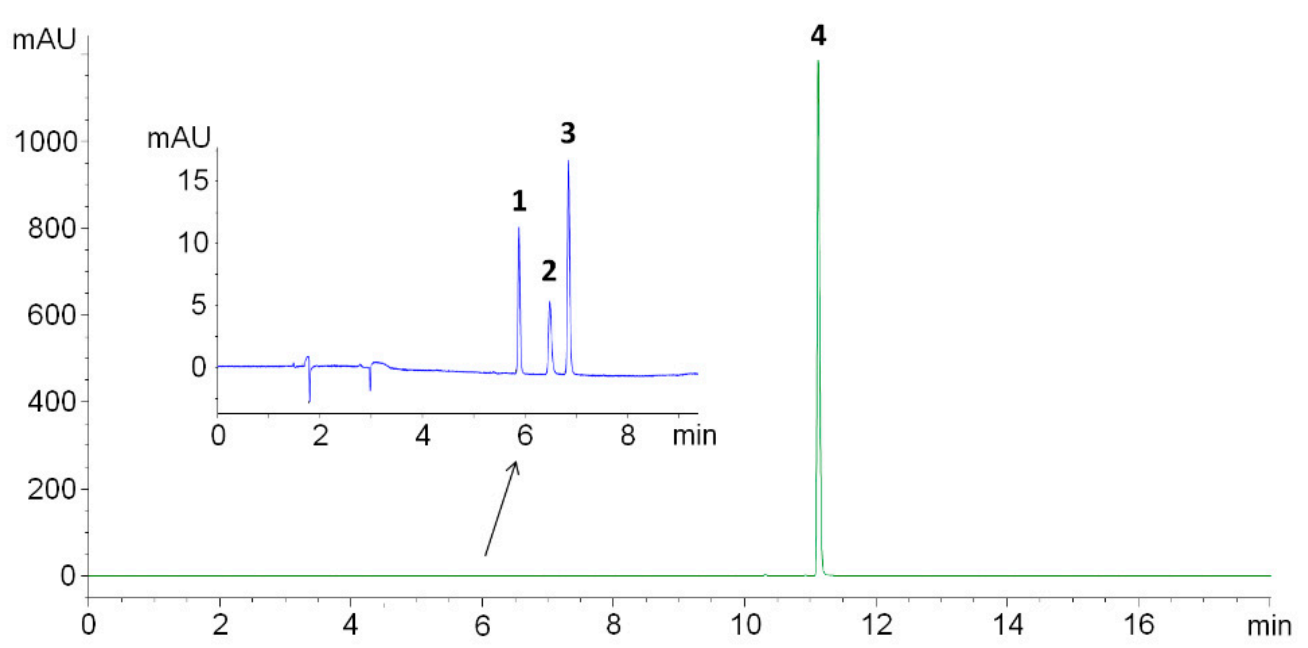

Figure 2. Chromatogram of a working solution showing FA (1), SA (2) and SP (3) at $285 \mathrm{~nm}$ (blue line) and SASP (4) at 360 nm (green line).

Table 1. Method validation data.

\begin{tabular}{|c|c|c|c|c|c|c|}
\hline Analyte & $\begin{array}{c}\text { Relative } \\
\text { Retention Time }\end{array}$ & $\begin{array}{l}\text { Linearity Range } \\
(\mu \mathrm{g} / \mathrm{mL})\end{array}$ & Regression Equation & $r$ & $\begin{array}{l}\text { Limit of Detection } \\
\qquad(\mu \mathrm{g} / \mathrm{mL})\end{array}$ & $\begin{array}{c}\text { Limit of } \\
\text { Quantification } \\
(\mu \mathrm{g} / \mathrm{mL})\end{array}$ \\
\hline SASP & 1.00 & $200-300$ & $y=15.12 x+373.40$ & 0.9999 & / & / \\
\hline FA & 0.53 & $0.4-0.6$ & $y=64.79 x-0.04$ & 0.9999 & 0.03 & 0.10 \\
\hline SA & 0.58 & $0.13-2.25$ & $y=19.02 x+0.14$ & 0.9997 & 0.04 & 0.13 \\
\hline \multirow[t]{2}{*}{$\mathrm{SP}$} & 0.62 & $0.13-2.25$ & $y=45.09 x-0.30$ & 0.9999 & 0.04 & 0.13 \\
\hline & \multicolumn{2}{|c|}{ Precision as RSD (\%) } & \multicolumn{4}{|c|}{ Accuracy as Recovery \pm RSD (\%) } \\
\hline Analyte & $\begin{array}{l}\text { Intra-Day } \\
\text { Precision } \\
(n=6)\end{array}$ & $\begin{array}{c}\text { Inter-Day } \\
\text { Precision } \\
(n=9)\end{array}$ & $\begin{array}{c}\text { Low } \\
(n=3)\end{array}$ & \multicolumn{2}{|c|}{$\begin{array}{l}\text { Medium } \\
\quad(n=3)\end{array}$} & $\begin{array}{l}\text { High } \\
(n=3)\end{array}$ \\
\hline SASP & 0.08 & 0.10 & $99.14 \pm 0.16$ & \multicolumn{2}{|c|}{$99.74 \pm 0.09$} & $99.27 \pm 0.16$ \\
\hline FA & 0.77 & 0.78 & $101.71 \pm 2.05$ & \multicolumn{2}{|c|}{$100.45 \pm 0.96$} & $101.54 \pm 0.76$ \\
\hline SA & 0.35 & 0.35 & $103.84 \pm 0.76$ & \multicolumn{2}{|c|}{$102.50 \pm 0.37$} & $102.25 \pm 0.46$ \\
\hline SP & 0.67 & 0.67 & $101.15 \pm 1.40$ & \multicolumn{2}{|c|}{$101.01 \pm 0.34$} & $99.59 \pm 1.01$ \\
\hline
\end{tabular}

Six individually prepared samples were analyzed within one day for determination of the intra-day precision, whilst for the inter-day precision, samples in triplicate were prepared and analyzed each day for three consecutive days. The low relative standard deviation (RSD) values of the intra-day $(\geq 0.77 \%)$ and inter-day $(\geq 0.78 \%)$ precisions implied that the sample preparation process and method remained precise within and between the days. The method accuracy at the three concentration levels was tested in triplicate and expressed as the recovery with accompanying RSD values. The accuracy for SASP and FA was tested in triplicate at $80 \%, 100 \%$ and $120 \%$ concentration levels of the working solution, whilst for SA and SP, the accuracy was tested at the limit of quantification as the lowest point, as well as at $100 \%$ and $180 \%$ of the working solution concentration. Satisfactory results were obtained for all analytes, ranging from $99.14 \% \pm 0.16 \%$ up to $103.84 \% \pm 0.76 \%$. The accuracy and precision data are shown in Table 1.

The method was shown to be robust regarding the recovery (assays remained within the regulatory limit of $100.0 \% \pm 5.0 \%)$, retention times $(\mathrm{RSD}<5 \%)$ and resolution $\left(R_{\mathrm{S}}>1.5\right)$ on the changes in the flow rate $(1.00 \pm 0.05 \mathrm{~mL} / \mathrm{min})$, column temperature $\left(25.0 \pm 1.0^{\circ} \mathrm{C}\right)$, mobile phase $\mathrm{pH}(5.0 \pm 0.1)$ and changes in the gradient $( \pm 1 \%$ of methanol). 


\subsection{TG/DTA Measurements}

The TG/DTA measurements of SASP and FA were done on a 1:1 binary mixture to increase the intensity of the possible reactions and for a better visualization of the incompatibility [19]. The TG/DTA curves of SASP, FA and the SASP/FA blend are shown in Figure 3. The DTA measurements of SASP showed an endothermic peak at $259.0^{\circ} \mathrm{C}$, which corresponded to its melting point, followed by degradation, which was observed as an exothermic peak. The TG measurement of SASP showed a weight loss of $2.0 \%$ in the temperature range of $0-100{ }^{\circ} \mathrm{C}$ due to the water evaporation from the sample. The weight remained constant until the beginning of the melting of SASP, followed by its degradation, resulting in a notable loss of $31.9 \%$ in the range of $240-300{ }^{\circ} \mathrm{C}$. With the further heating of the sample, a constant weight loss was observed in the temperature range of $300-400{ }^{\circ} \mathrm{C}$, with the final weight of the sample being $55.3 \%$ of the starting value (Figure $3 a$ ).
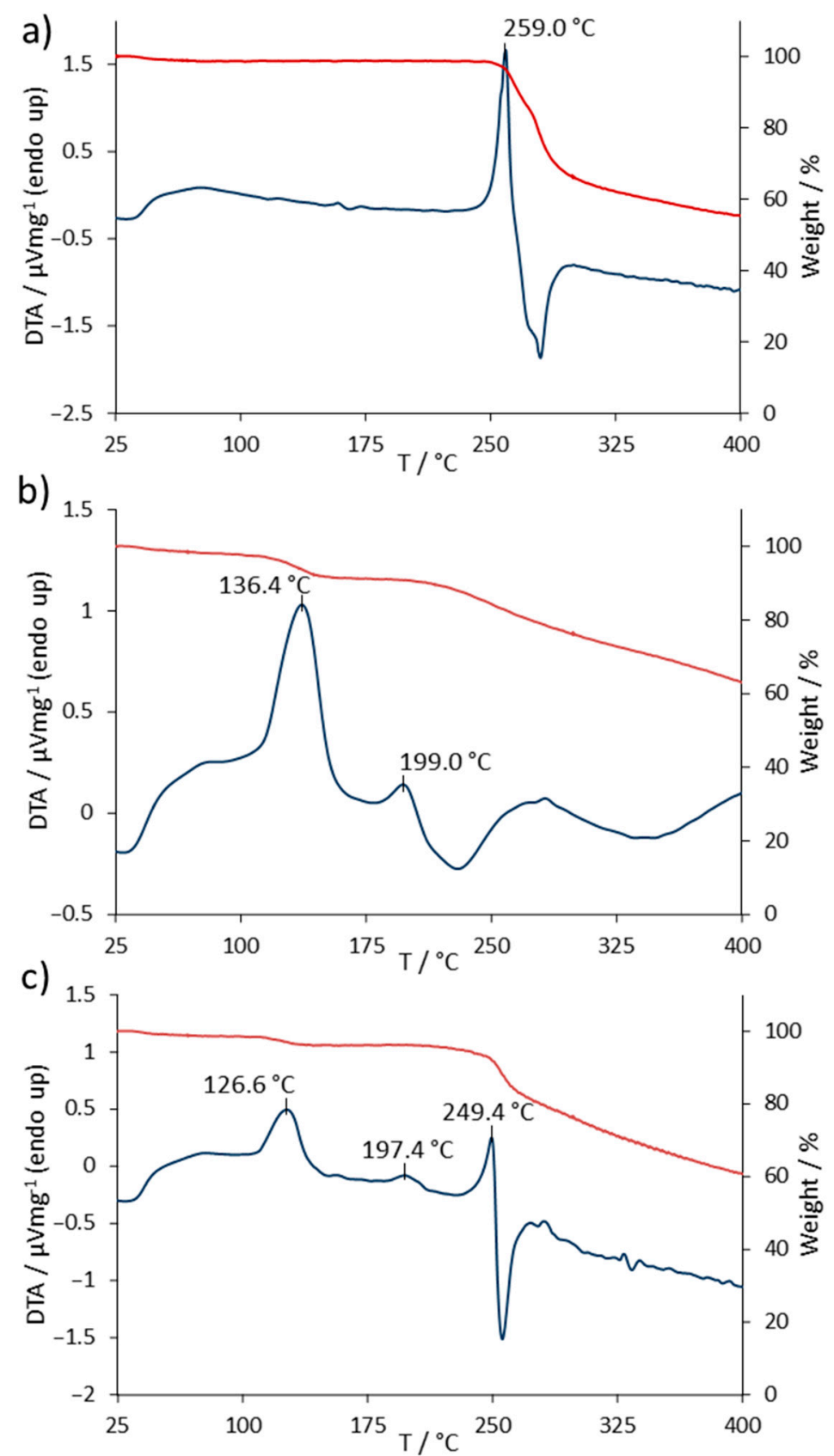

Figure 3. Thermogravimetry (TG) (red line) and differential thermal analysis (DTA) (blue line) curves of (a) SASP, (b) FA and (c) SASP/FA 1:1 blend. 
The DTA measurements of FA showed two endothermic peaks at 136.4 and $199.0{ }^{\circ} \mathrm{C}$. The peak at $136.4^{\circ} \mathrm{C}$ corresponded to the endothermic nature of the water evaporation process. The TG measurements in the range of $0-175{ }^{\circ} \mathrm{C}$ showed a weight loss of $8.4 \%$, which suited the declared value of the water content in the used FA standard of $8.0 \%$ [20]. The endothermic peak at $199.0^{\circ} \mathrm{C}$ corresponded to the FA melting temperature, followed by a slow degradation as the heating continued, which could be seen as a constant loss of weight in the range of $200-400{ }^{\circ} \mathrm{C}$ [12]. The weight of the FA sample upon the end of measurements was reduced to $63 \%$ of its starting weight (Figure $3 b$ ).

The TG/DTA measurements of the SASP/FA blend showed both SASP and FA previously observed characteristic peaks. The endothermic peak at $126{ }^{\circ} \mathrm{C}$ corresponded to the loss of water present mainly in the FA standard. A weight loss of $3.9 \%$ was observed, which was equivalent to that of $8.4 \%$ observed in the FA standard alone, since the SASP/FA blend was prepared in a 1:1 ratio. The FA characteristic melting temperature was observed at $197.4^{\circ} \mathrm{C}$, whilst a slight shift in the SASP endothermic peak occurred, resulting in a temperature of $249.4^{\circ} \mathrm{C}$. The final weight of the sample was $60.8 \%$ of its starting weight, which fell in between the observed final weights of the SASP and FA standards, implying that no additional loss in combination was observed (Figure 3c).

The incompatibility of two drugs can result in the change of their physical characteristics, which can be observed as a shift in the characteristic peak temperature $\left( \pm 5^{\circ} \mathrm{C}\right)$ or if the peak notably changes its shape, disappears or a new peak occurs $[12,19,21,22]$. In the provided measurements, a shift of the peak temperature was observed for SASP $\left(9.6^{\circ} \mathrm{C}\right)$; on the other hand, the FA characteristic melting peak did not shift notably $\left(1.6^{\circ} \mathrm{C}\right)$, which implies that the two drugs might be compatible. Even though the peak shapes remained intact and there was no occurrence of new peaks, the shift in the SASP peak was above the tolerance described in the literature, which might indicate possible changes in the system. However, it is well-known that conclusions cannot be made based only on TG/DTA measurements, since they provide limited information, and the use of other complementary analytical methods is mandatory to get a better understanding of the possible interactions [11,23].

\subsection{IST Followed by FTIR and HPLC Measurements}

In isothermal stress testing, the samples are subjected to isothermal conditions for a longer period, usually two-six weeks, to get an insight into the possible interactions between two or more components in the blend. FTIR is commonly used for getting an insight into the possible chemical changes in the sample, indicating the creation of new bonds, which ultimately results in different FTIR spectra. The FTIR spectra of SASP, FA and their blends are shown in Figure 4.

The obtained spectra show most of the SASP and FA characteristic absorption bands; however, since SASP and FA are molecules with numerous bonds, the FTIR spectra of the physical mixtures are hard to interpret. The SASP characteristic bands $(\mathrm{C}=\mathrm{O}$ stretching from the carboxyl group at $1674 \mathrm{~cm}^{-1}, \mathrm{~S}=\mathrm{O}$ from sulphonamide at $1356 \mathrm{~cm}^{-1}$ and $\mathrm{C}-\mathrm{O}$ stretching at $1078 \mathrm{~cm}^{-1}$ ) are more pronounced in the 500:1, 10:1, 5:1 and 2:1 blends; however, as the ratio of SASP and FA equalized (1:1), the FA characteristic bands $(\mathrm{C}=\mathrm{O}$ stretching at $1687 \mathrm{~cm}^{-1}$ and $\mathrm{N}-\mathrm{H}$ bending at $1602 \mathrm{~cm}^{-1}$ ) appeared and became more dominant as the FA ratio in the blends increased (1:2, 1:5 and 1:10).

The interpretation of the FTIR spectra of the physical mixtures may lead to false conclusions due to the presence of the overlapping bands. Therefore, without going into a deeper visual analysis of the obtained spectra, where, as said above, we cannot gain an unambiguous conclusion, we decided to introduce a statistical approach with the aim of a better interpretation of the obtained spectra. Multivariate statistical analysis tools, such as PCA and CA, were already used and are well-described in the literature for the interpretation of obtained data [24,25]. 


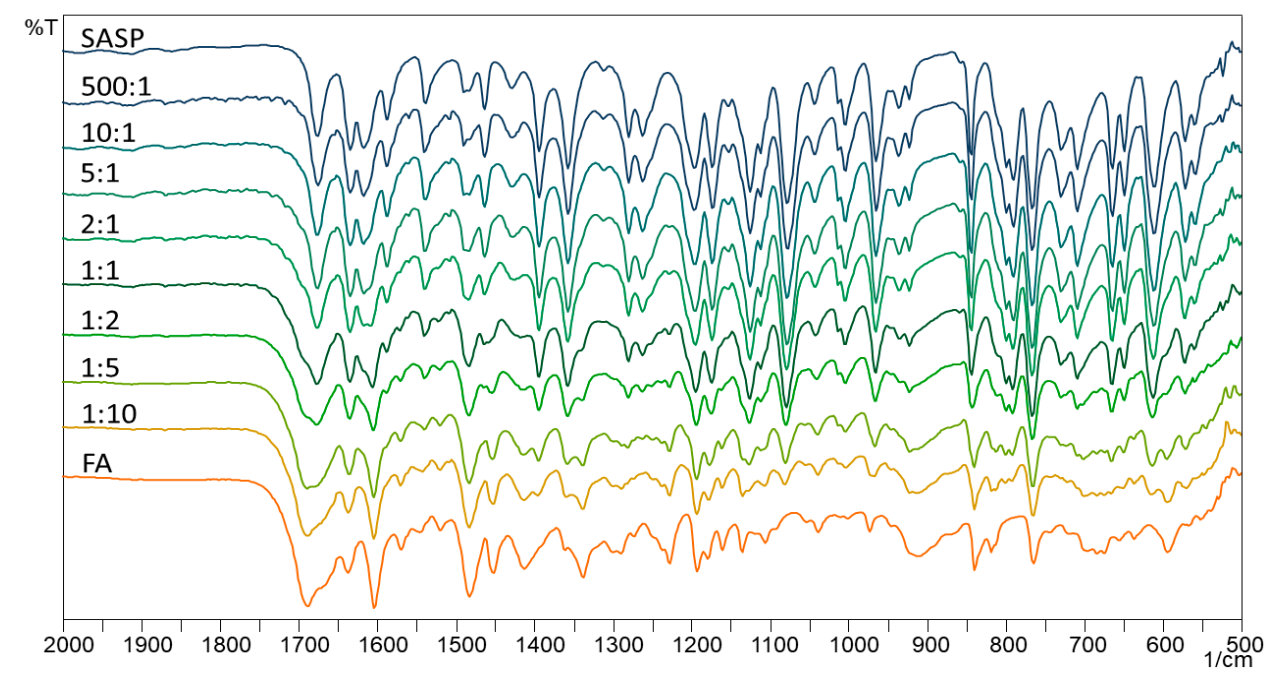

Figure 4. Fourier-transform infrared (FTIR) spectra of SASP, FA and their blends.

The results of the PCA analysis showed that eigenvalues of the nine obtained eigenvectors ranged from 494.6 to 0.2 , whilst the data variability was in range from $76.21 \%$ to $0.03 \%$ (Table 2).

Table 2. Eigenvalues and variances of the nine obtained principal components.

\begin{tabular}{ccccccccccc}
\hline Eigenvector & $\mathbf{1}$ & $\mathbf{2}$ & $\mathbf{3}$ & $\mathbf{4}$ & $\mathbf{5}$ & $\mathbf{6}$ & $\mathbf{7}$ & $\mathbf{8}$ & $\mathbf{9}$ \\
\hline Eigenvalue & 494.6 & 134.8 & 7.6 & 6.2 & 2.4 & 1.9 & 1.0 & 0.4 & 0.2 \\
Variability (\%) & 76.21 & 20.77 & 1.17 & 0.95 & 0.37 & 0.29 & 0.15 & 0.07 & 0.03 \\
Cumulative (\%) & 76.21 & 96.98 & 98.15 & 99.10 & 99.47 & 99.76 & 99.91 & 99.97 & 100.00 \\
\hline
\end{tabular}

The eigenvector with the greatest variability and eigenvalue was the most impactful principal component, implying that the eigenvector with the eigenvalue of 494.6 and a variability of $76.21 \%$ was the first principal component (PC1). Since $96.98 \%$ of the total variance was covered by the first two eigenvectors (PC1 and PC2), the eigenvalues and variabilities of the last seven eigenvectors (three-nine) were insignificant and, therefore, were excluded from further analyses.

For a better visualization of the obtained principal components and data variability, the PCA results were best visualized using a PC1 vs. PC2 bidimensional scatterplot (Figure 5).

FA is located far to the right with the most positive PC1 value (41.33), whilst SASP is located far to the left with the most negative PC1 value $(-27.49)$, where the blends of SASP and FA are placed in an increasing PC1 order as the SASP ratio in the blends reduces and the FA ratio increases (Figure 5a). These results are in favor of the SASP and FA chemical compatibility, showing the similarity between the spectra of the two adjacent blends. Random placement of the samples along the PC1 axis would indicate that there is no similarity between the obtained spectra due to the possible chemical reaction between the components in the blends.

The cluster analysis (Figure 5b) precisely reflected the results present in the scatterplot. Two main clusters were formed; the first connected SASP and the blends with a higher SASP share (500:1, 10:1, 5:1 and 2:1), whilst the other connected FA and its blends with a higher FA share (1:10, 1:5 and 1:2). The SASP/FA 1:1 blend was placed in a cluster with stronger linkage to the SASP-dominant blends, since the SASP spectrum overlapped most of the FA characteristic bands. The dissimilarity values between the formed clusters were below the strong Sokai and Sneath distance criteria (33\% of the maximum distance, dashed line), which indicated that there was a statistically significant similarity between the obtained spectra, therefore implying that no chemical reaction occurred in the blends. 

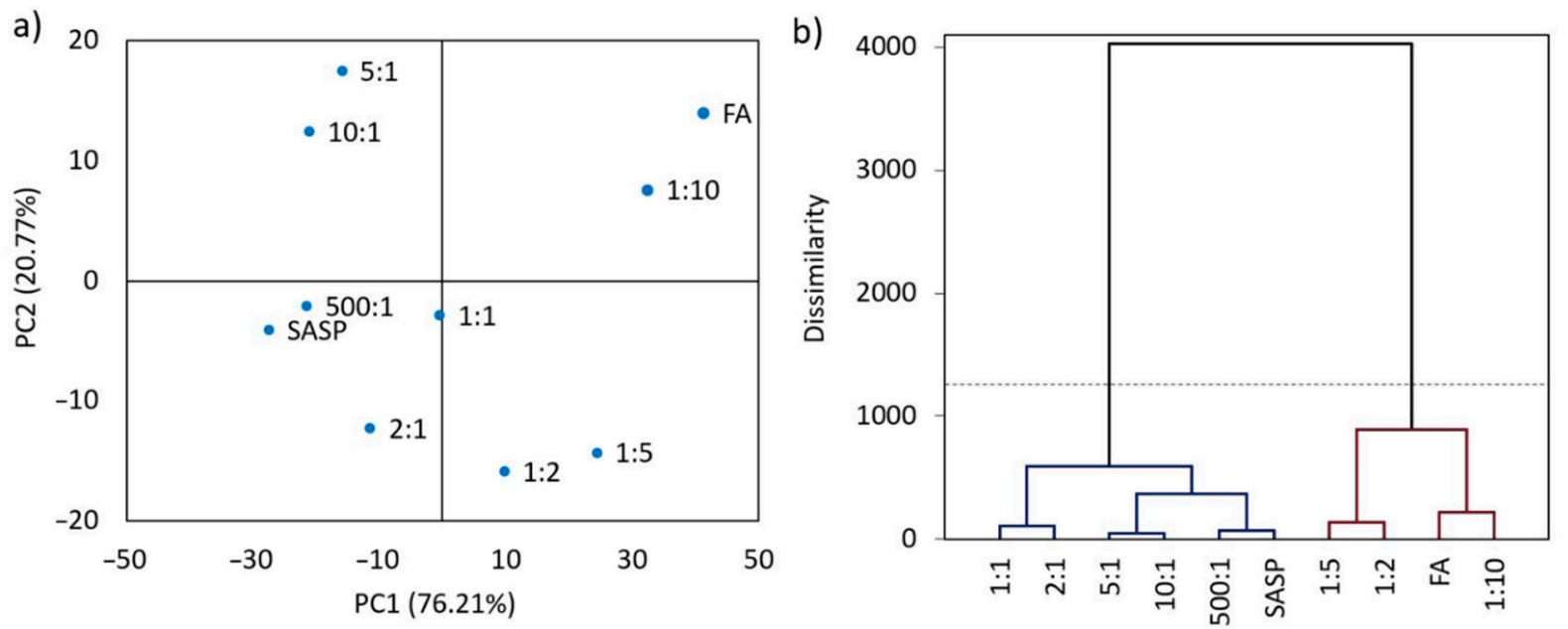

Figure 5. Results of the statistical analysis showing (a) the principle component analysis (PCA) bidimensional scatterplot and (b) cluster analysis (CA) dendrogram, indicating similarities between SASP, FA and their blends.

The IST is usually followed by HPLC measurements to determine any assay drops as a result of drug-drug interactions. Table 3 shows the results of the chromatographic analysis, as well as the visual inspection observations.

Table 3. Results of the chromatographic analysis after isothermal stress testing (IST).

\begin{tabular}{|c|c|c|c|c|}
\hline API & Sample Type & Appearance & Physical Change & $\begin{array}{c}\text { Recovery } \pm \text { RSD (\%) } \\
(n=3)\end{array}$ \\
\hline SASP & $\begin{array}{c}\text { standard } \\
\text { tablet } \\
\text { standards blend } \\
\text { tablets blend }\end{array}$ & brownish-yellow powder & $\begin{array}{c}\text { no significant visual } \\
\text { changes }\end{array}$ & $\begin{array}{c}99.0 \pm 0.5 \\
102.5 \pm 1.3 \\
99.9 \pm 2.1 \\
101.0 \pm 1.2\end{array}$ \\
\hline FA & $\begin{array}{c}\text { standard } \\
\text { tablet } \\
\text { standards blend } \\
\text { tablets blend }\end{array}$ & $\begin{array}{c}\text { yellow powder } \\
\text { pale yellow powder } \\
\text { brownish-yellow powder } \\
\text { brownish-yellow powder }\end{array}$ & $\begin{array}{c}\text { no significant visual } \\
\text { changes }\end{array}$ & $\begin{array}{l}98.4 \pm 0.6 \\
99.3 \pm 1.0 \\
98.6 \pm 1.6 \\
\quad \pm 2.7\end{array}$ \\
\hline
\end{tabular}

The HPLC analysis results showed a great stability under the imposed conditions, with little to no change in the determined assays. With the statistical analysis, using the twosample $t$-test, it was determined that the recovered assays were not significantly different $(t$-value $=0.72, t$-critical $=3.18, p$-value $=0.52, \alpha<0.05)$ in the stressed blends from those when stressed alone.

\subsection{Forced Degradation Study}

Forced degradation studies were applied on SASP and FA standards, standards blend and tablets blend to get an insight into the degradation behavior of each component when stressed alone and in the presence of each other. The goal of this approach was to examine the chemical stability of SASP and FA and to determine whether they impacted each other's stability in the applied conditions. Samples were exposed to alkali, acid, oxidative, thermal and photolytic conditions and kept in the same until the optimal degradation of $10-30 \%$. Keeping the samples too long in defined conditions would result in extensive degradation and the formation of unwanted secondary or tertiary degradation products, which could misslead the experiment; however, if the analyte shows little to no degradation in the seven-day period, it can be stated that the substance is stable in the applied conditions. The optimal degradation time used for stressing of the blends was the time needed for the first standard to degrade in the desired range when stressed individually; therefore, using that same reaction time for both blends' comparable results can be obtained regarding 
the degradation of that component. Stressed samples were analyzed using the developed HPLC method to determine the assay drops, as well as occurrence of SASP-related impurities of SA and SP. Degradation times and assay losses of stressed samples are presented in Table 4.

Table 4. Forced degradation study results.

\begin{tabular}{|c|c|c|c|c|c|c|c|}
\hline \multirow{3}{*}{ Degradation Condition } & \multirow{3}{*}{ Degradation Times/day ${ }^{1}$} & \multicolumn{6}{|c|}{ Observed Drops of Assay/\% } \\
\hline & & \multicolumn{2}{|c|}{ Standards } & \multicolumn{2}{|c|}{ Standards Blend } & \multicolumn{2}{|c|}{ Tablets Blend } \\
\hline & & SASP & FA & SASP & FA & SASP & FA \\
\hline $0.1 \mathrm{M} \mathrm{NaOH}$ (solution) & 7 & 1.8 & 2.7 & 0.8 & 2.7 & 2.3 & 2.4 \\
\hline $0.1 \mathrm{M} \mathrm{HCl}$ (solution) & $7(1)$ & 0.8 & 21.6 & 0.4 & 17.4 & 1.7 & 19.0 \\
\hline $3 \% \mathrm{H}_{2} \mathrm{O}_{2}$ (solution) & $7(1)$ & 1.7 & 11.7 & n.d. ${ }^{2}$ & 11.8 & 2.2 & 10.4 \\
\hline Photo (solution) & $7(1)$ & 1.5 & 10.0 & 0.3 & 14.6 & 1.6 & 16.7 \\
\hline Photo (solid) & 7 & 1.7 & 2.9 & 1.3 & 0.6 & 1.6 & 3.7 \\
\hline Thermal (solution) & 7 & 1.9 & 1.1 & n.d. & 100.0 & n.d. & 100.0 \\
\hline Thermal (solid) & 7 & 1.9 & 1.0 & 2.4 & 1.8 & 0.2 & 2.2 \\
\hline
\end{tabular}

${ }^{1}$ Values in the parentheses represent the optimal degradation time for the FA standard and FA in the prepared blends. ${ }^{2}$ n.d. $=$ no degradation observed.

As can be seen from the results, the SASP standard remained stable in all conditions for 7 days, with the maximum assay drop of $1.9 \%$ observed in thermally stressed solution. FA solution was shown to be stable in alkali and thermal conditions for 7 days with the assay drop not exceeding $2.7 \%$. Solid samples of FA exposed to thermal and photolytic conditions for 7 days measured assay drop of not more than $2.9 \%$, implying stability in those conditions. FA solution was shown to undergo degradation in acid, peroxide and photolytic conditions, after 1 day, resulting in assay drops of $21.6 \%, 11.7 \%$ and $10.0 \%$, respectively. SASP remained stable when combined with FA in prepared standard and tablet blends through 7 (or 1) day exposure to all conditions, with the maximum degradation of $2.4 \%$. FA in blends has shown similar stability in all stress conditions except when heat-stressed as standard and tablet blend solution, where severe degradation occurred with FA assay drop of $100 \%$ after 1 day, compared to $1.1 \%$ degradation after 7 days as standard solution. Considering that FA is highly unstable in low $\mathrm{pH}$ environment, especially at elevated temperatures [26], as it was observed in our study (17.4-21.6\% assay drops at room temperature and $100 \%$ at $70{ }^{\circ} \mathrm{C}$ ), this phenomena can be justified with the fact that prepared $250 \mu \mathrm{g} / \mathrm{mL}$ SASP solution in $30 \%$ DMF measures $\mathrm{pH}$ of 2.85 (compared to pure $30 \%$ DMF solution that measures $\mathrm{pH}$ of 6.35), creating the acidic environment in which FA undergoes degradation, whilst heating of the sample significantly increases reaction kinetics, which ultimately resulted in complete FA degradation in a short period. Considering the absence of SASP/FA interactions observed with the previous methods, it can be said that the observed drop in FA assay is strictly $\mathrm{pH}$ \& temperature related, rather than as a result of chemical interaction between SASP and FA. The impact of $\mathrm{pH}$ on instability of FA as well as the impact of $\mathrm{pH}$ in combination with elevated temperatures is well described in the literature [27-30]. However, given that SASP is responsible for creating a low $\mathrm{pH}$ environment when dissolved, this must be taken into consideration during the formulation process. In all degradations conducted SASP related impurities SP and SA were not detected. Purity of SASP and FA chromatographic peaks remained higher than 998 after every degradation conducted (purity threshold set to 995 whilst purity value of 1000 represents the identical spectra).

\subsection{Drug Dissolution Testing}

The impact of drugs on their release and stability can be examined with in-vitro dissolution studies using simulated gastric and intestinal fluids [10,12,31,32]. Drug release and stability were tested according to USP procedure using the two-stage dissolution process. The first stage concerned 120-min dissolution of tablets in $0.1 \mathrm{~N} \mathrm{HCl}$ solution, 
also known as USP simulated gastric fluid. The second stage involved 60-min dissolution in $0.05 \mathrm{mM}$ PB with $\mathrm{pH}$ set to 7.5, also known as USP simulated intestinal fluid. A twostage dissolution process is necessary since the tested SASP product comes in a form of gastro-resistant tablets, securing that most of the drug is released in the colon, rather than in the stomach.

As expected, SASP was not released from the formulation during the first two hours of the first dissolution stage, followed by the second stage with the gradual release of SASP over one hour resulting in a total release of $100.7 \% \pm 0.8 \%$. The effect of FA on the SASP release was tested by placing the SASP and FA tablets in the dissolution vessel at the beginning of the experiment to test the impact of dissolved FA formulation on the gastro-resistant film of the SASP tablet, which could result in premature release of SASP from the formulation. In the two-hour acid stage, no SASP was released from the formulation whilst in the following stage release of $98.8 \% \pm 0.4 \%$ was observed. Both SASP alone and SASP in the presence of FA gave similar release curves (Figure 6a), which can be confirmed by calculating the difference $\left(f_{1}\right)$ and similarity factors $\left(f_{2}\right)$ [33]. The calculated difference factor of $1.6 \%$ is well below the maximum limit of $15.0 \%$, whilst a high similarity factor value of $92.4 \%$ implies great similarity, considering that the $100.0 \%$ similarity value corresponds to two identical curves.

On the other hand, FA showed a gradual release in simulated gastric fluid, resulting in the recovery of $77.1 \% \pm 4.9 \%$ during the first two hours, followed by the release of $109.7 \% \pm 0.6 \%$ in the second dissolution stage. The possible occurrence of incompatibility between FA and SASP, considering the previously observed degradation in the forced degradation study, was tested by placing both tablets in the dissolution vessel at the beginning of the experiment. In both the acid and buffer stages, FA showed similar release as when tested alone, with the recovery values of $72.3 \% \pm 4.0 \%$ and $107.2 \% \pm 2.2 \%$, respectively (Figure 6b). Difference $\left(f_{1}\right)$ and similarity factors $\left(f_{2}\right)$ were calculated as well with obtained values of 3.01 and 77.91, respectively, implying that the obtained curves were similar, and no significant interaction between two drugs occurred. 

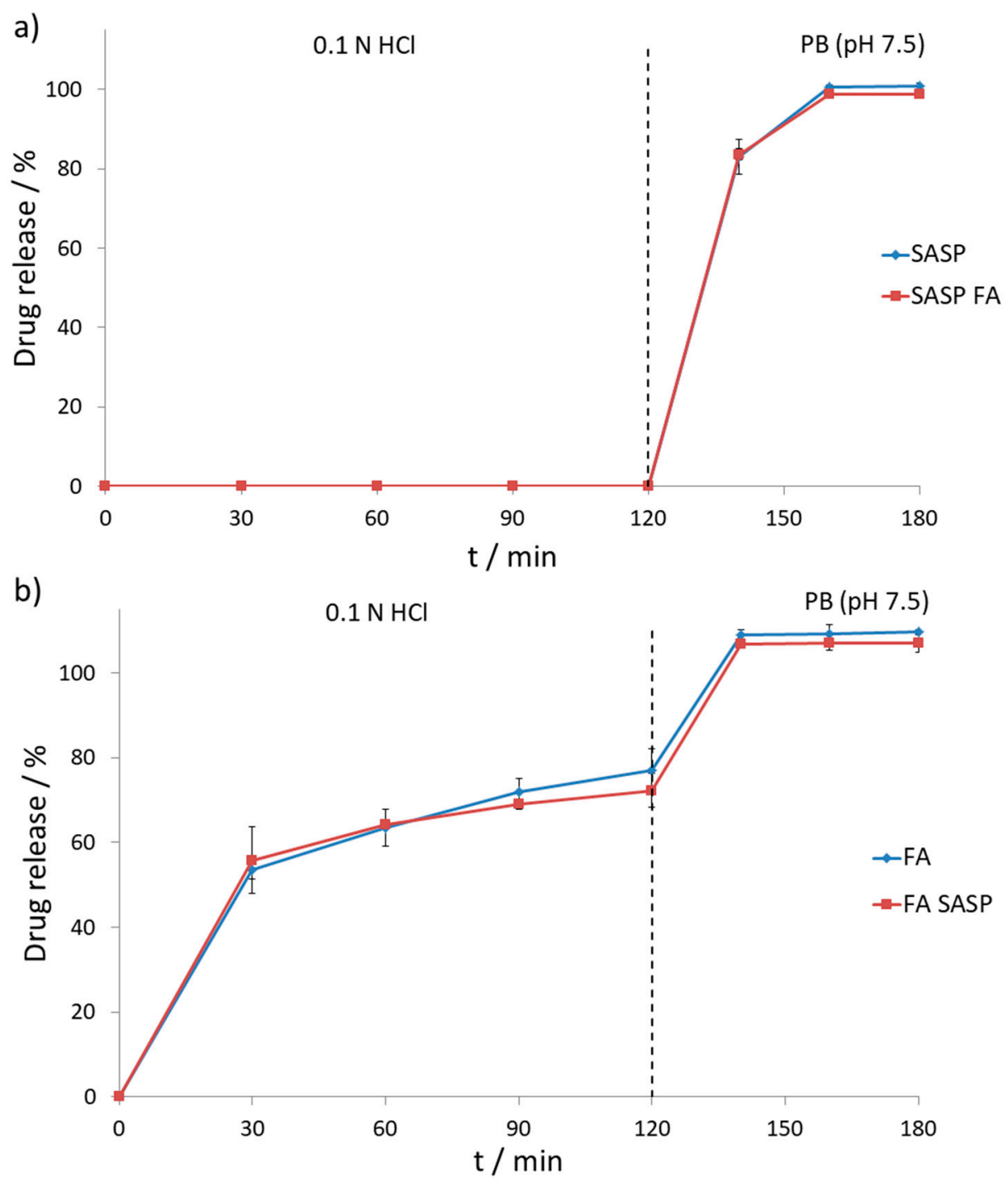

Figure 6. Drug release profiles of (a) SASP and (b) FA alone (blue line) and in combination (red line) in different dissolution media $(n=3)$ (Dashed line represents the change of dissolution media).

\section{Conclusions}

SASP and FA physicochemical compatibility was successfully examined using various analytical techniques. DTA measurements showed a shift of the SASP peak when present in a blend with FA; however, no changes of the FA peak and no occurrence of new peaks nor the disappearance of existing peaks were observed. The TG measurements showed no significant changes in weight loss, which indicated a lack of interactions; however, conclusions cannot be made solely on the TG/DTA measurements. The FTIR measurements in combination with the PCA/CA analysis implied that no interaction occurred between SASP and FA, which was also confirmed with the chromatographic analysis of the samples after IST, forced degradation and dissolution studies. Considering the ratio in which the SASP and FA samples were prepared (500:1), a significant loss of the assay of FA could be observed if any chemical interaction occurred. The thermal stress study of the SASP/FA standard and tablet solution resulted in the complete degradation of FA; however, it was explained as a $\mathrm{pH}$ - and temperature-related process and not a result of the SASP and FA interaction. Although the TG/DTA measurements raised concerns, with the wider picture obtained using various analytical tools, this work implies that SASP and FA are physicochemically compatible. This work presents one segment of the FDC development process; however, further studies, such as selecting optimal excipients, drug-excipient 
compatibility studies and formulation stability studies, as well as dissolution studies of prepared dosage units, should be conducted to create an acceptable product.

Author Contributions: Conceptualization, M.-L.J., A.M. and E.B.; methodology, M.-L.J., E.B., S.K. and M.C.; software, M.-L.J. and M.C.; validation, M.-L.J.; formal analysis, M.-L.J. and E.B.; investigation, M.-L.J. and E.B.; resources, A.M.; data curation, M.-L.J. and E.B.; writing-original draft preparation, M.-L.J. and A.M.; writing-review and editing, M.-L.J., E.B., S.K., D.A.K., B.N. and A.M.; visualization, M.-L.J.; supervision, A.M. and B.N.; project administration, A.M. and funding acquisition, A.M. All authors have read and agreed to the published version of the manuscript.

Funding: This research was funded by Croatian Science Foundation, grant numbers HRZZ-UIP2017-05-3949 and HRZZ-DOK-2018-01-9047.

Institutional Review Board Statement: Not applicable.

Informed Consent Statement: Not applicable.

Data Availability Statement: The data presented in this study are available on request from the corresponding author. The data are not publicly available due to privacy or ethical restrictions.

Conflicts of Interest: The authors declare no conflict of interest.

\section{References}

1. Mulder, D.J.; Noble, A.J.; Justinich, C.J.; Duffin, J.M. A tale of two diseases: The history of inflammatory bowel disease. J. Crohn's Coliti 2014, 8, 341-348. [CrossRef] [PubMed]

2. Taffet, S.L.; Das, K.M. Sulfasalazine-Adverse effects and desensitization. Dig. Dis. Sci. 1983, 28, 833-842. [CrossRef]

3. Jansen, G.; Van Der Heijden, J.; Oerlemans, R.; Lems, W.F.; Ifergan, I.; Scheper, R.J.; Assaraf, Y.G.; Dijkmans, B.A.C. Sulfasalazine is a potent inhibitor of the reduced folate carrier: Implications for combination therapies with methotrexate in rheumatoid arthritis. Arthritis Rheum. 2004, 50, 2130-2139. [CrossRef] [PubMed]

4. Biasco, G.; Di Marco, M.C. Folate and prevention of colorectal cancer in ulcerative colitis. Eur. J. Cancer Prev. 2005, 14, 395-398. [CrossRef] [PubMed]

5. Gomollón, F.; Gisbert, J.P. Anemia and inflammatory bowel diseases. World J. Gastroenterol. 2009, 15, 4659-4665. [CrossRef]

6. Safi, J.; Joyeux, L.; Chalouhi, G.E. Periconceptional Folate Deficiency and Implications in Neural Tube Defects. J. Pregnancy 2012, 2012, 1-9. [CrossRef]

7. Kubota-Sjogren, Y.; Harding, K.; Irving, P.; Nelson-Piercy, C. Inflammatory bowel disease in pregnancy: Management strategy based on best evidence and European guidelines. Br. J. Gen. Pr. 2014, 64, 593-594. [CrossRef]

8. Begum, S.G.; Reddy, Y.D.; Divya, B.S.; Komali, P.; Sushmitha, K.; Ruksar, S. Pharmaceutical Incompatibilites: A Review. Asian J. Pharm. Res. Dev. 2018, 6, 56-61. [CrossRef]

9. Chidambaram, M.; Krishnasamy, K. Drug-Drug/Drug-Excipient Compatibility Studies on Curcumin using Non-Thermal Methods. Adv. Pharm. Bull. 2014, 4, 309-312.

10. Petruševska, V.; Lasić, K.; Mornar, A. Compatibility investigation for a new antituberculotic fixed dose combination with an adequate drug delivery. Drug Dev. Ind. Pharm. 2020, 46, 1-30. [CrossRef] [PubMed]

11. Lavor, E.P.; Navarro, M.V.M.; Freire, F.D.; Aragão, C.F.S.; Raffin, F.N.; Barbosa, E.G.; Moura, T.F.A.D.L.E. Application of thermal analysis to the study of antituberculosis drugs-excipient compatibility. J. Therm. Anal. Calorim. 2014, 115, 2303-2309. [CrossRef]

12. Jeličić, M.-L.; Brusač, E.; Amidžić Klarić, D.; Nigović, B.; Keser, S.; Mornar, A. Physicochemical Compatibility Investigation of Mesalazine and Folic Acid Using Chromatographic and Thermoanalytical Techniques. Pharmaceuticals 2020, 13, 187. [CrossRef]

13. World Health Organization Guidelines for Registration of Fixed-Dose Combination Medicinal Products. Available online: https: //www.who.int/medicines/areas/quality_safety/quality_assurance/GuidelinesRegistrationFixedDoseCombinationTRS929 Annex5.pdf (accessed on 15 January 2021).

14. British Pharmacopoeia, 2020 ed.; Medicines and Healthcare Products Regulatory Agency: London, UK, 2020.

15. Rowe, R.C.; Sheskey, P.J.; Quinn, M.E. Handbook of Pharmaceutical Excipients, 6th ed.; Pharmaceutical Press: London, UK, 2009.

16. The United States Pharmacopeia. The National Formulary; United States Pharmacopoeial Convention: Rockville, MD, USA, 2014; Volume 3.

17. Hryniewicka, M.; Starczewska, B.; Gołębiewska, A. Determination of Budesonide and Sulfasalazine in Water and Wastewater Samples Using DLLME-SFO-HPLC-UV Method. Water 2019, 11, 1581. [CrossRef]

18. Validation of Analytical Procedures: Text and Methodology Q2(R1). Available online: https://database.ich.org/sites/default/ files/Q2\%28R1\%29Guideline.pdf (accessed on 15 January 2021).

19. Dourado, D. Thermal Analysis as a Useful Tool in Drug-Excipient Compatibilty Studies: The Impact in Pharmaceuticals Products. Biomed. J. Sci. Tech. Res. 2019, 22, 16634-16636. [CrossRef] 
20. Sigma-Aldrich Certificate of Analysis for Folic Acid-Meets USP Testing Specifications, Product no. F8798, Batch no. SLBH7728V. Available online: https://www.sigmaaldrich.com/catalog/CertOfAnalysisPage.do?symbol=F8798\&LotNo=SLBH7728V\& brandTest=SIAL\&returnUrl=\%2Fproduct\%2FSIAL\%2FF8798 (accessed on 28 September 2020).

21. Rojek, B.; Wesolowski, M. DSC supported by factor analysis as a reliable tool for compatibility study in pharmaceutical mixtures. J. Therm. Anal. Calorim. 2019, 138, 4531-4539. [CrossRef]

22. Leyva-Porras, C.; Cruz-Alcantar, P.; Espinosa-Solís, V.; Martínez-Guerra, E.; Balderrama, C.I.P.; Martínez, I.C.; Saavedra-Leos, M.Z. Application of Differential Scanning Calorimetry (DSC) and Modulated Differential Scanning Calorimetry (MDSC) in Food and Drug Industries. Polymers 2019, 12, 5. [CrossRef]

23. Chadha, R.; Bhandari, S. Drug-excipient compatibility screening-Role of thermoanalytical and spectroscopic techniques. J. Pharm. Biomed. Anal. 2014, 87, 82-97. [CrossRef] [PubMed]

24. Rojek, B.; Wesolowski, M.; Suchacz, B. Detection of compatibility between baclofen and excipients with aid of infrared spectroscopy and chemometry. Spectrochim. Acta Part A: Mol. Biomol. Spectrosc. 2013, 116, 532-538. [CrossRef]

25. Rojek, B.; Wesolowski, M. Fourier transform infrared spectroscopy supported by multivariate statistics in compatibility study of atenolol with excipients. Vib. Spectrosc. 2016, 86, 190-197. [CrossRef]

26. Gazzali, A.M.; Lobry, M.; Colombeau, L.; Acherar, S.; Azaïs, H.; Mordon, S.; Arnoux, P.; Baros, F.; Vanderesse, R.; Frochot, C. Stability of folic acid under several parameters. Eur. J. Pharm. Sci. 2016, 93, 419-430. [CrossRef] [PubMed]

27. Daniel, E.P.; Kline, O. Factors affecting folic acid determination. J. Biol. Chem. 1947, 170, 739-746. [CrossRef]

28. Dick, M.; Harrison, I.; Farrer, K. The thermal stability of folic acid. Aust. J. Exp. Biol. Med. Sci. 1948, 26, 239-244. [CrossRef]

29. Paine-Wilson, B.; Chen, T.-S. Thermal destruction of folacin: Effect of pH and buffer ions. J. Food Sci. 1979, 44, 717-722. [CrossRef]

30. Liang, X.S.; Zhao, F.Q.; Hao, L.X. Research on Stability of Synthetic Folic Acid. Adv. Mater. Res. 2013, 781-784, 1215-1218. [CrossRef]

31. Jindal, K.; Chaudhary, R.; Singla, A.; Gangwal, S.; Khanna, S. Dissolution test method for rifampicin-isoniazid fixed dose formulations. J. Pharm. Biomed. Anal. 1994, 12, 493-497. [CrossRef]

32. Shishoo, C.; Shah, S.; Rathod, I.; Savale, S.; Kotecha, J.; Shah, P. Stability of rifampicin in dissolution medium in presence of isoniazid. Int. J. Pharm. 1999, 190, 109-123. [CrossRef]

33. Shah, V.P.; Tsong, Y.; Sathe, P.; Williams, R.L. Dissolution Profile Comparison Using Similarity Factor, f2. Dissolution Technol. 1999, 6, 15. [CrossRef] 